\title{
Experimental Investigations on Performance Comparison of EKF and ANN Based Controllers with UKF Based Controller for Autonomous Hybrid Systems with Uncertainties
}

\begin{abstract}
Shijoh Vellayikot ${ }^{\dagger}$ and M. V. Vaidyan*
Abstract - This paper proposes autonomous hybrid system (AHS) with estimator based inverse dynamics controller along with extended kalman filter and artificial neural network based state estimators ensuring best performance and robustness by minimum ISE in controlling non-measurable state variables of autonomous hybrid systems. With the help of experimental setup of benchmark model of AHS (hybrid three-tank system), the detailed performance comparison of these proposed methods was made both qualitatively and quantitatively with that of existing method (AHS with unscented kalman filter and inverse dynamics controller (UKFC)) under different operating conditions such as servo and regulatory operations, process-model parameter uncertainties, initial condition mismatch, and different types of faults in the system, subsequently the results are reported.
\end{abstract}

Keywords: Autonomous hybrid systems, State estimation, Estimator based controllers, Extended kalman filter, Unscented kalman filter, Artificial neural network

\section{Introduction}

When continuous and discrete behaviour interact each other in a process, the concept of hybrid systems arises [13]. With the introduction of computers for instrumentation and control in process industry, computer controlled systems, which can be considered as hybrid dynamic systems and where the discrete events in computer interact with the continuous systems and consequently the modeling, estimation, and control problems of hybrid systems demand more attention from the researchers in the present scenario. If the discrete variables of hybrid systems depend only on its continuous state variables, and not on any external inputs, then such systems are termed as autonomous hybrid systems (AHS) [3]. A hybrid threetank system was used as the benchmark example in many of the works [4-7] for demonstrating different modeling, estimation and control algorithms for hybrid systems and to a class of its subsystem like AHS. In this direction, experimental validation reported in [8] and [9] for the state estimation and the control of hybrid systems, has significance.

Finding optimum and robust state estimation schemes, and hence utilization of such estimators in many advanced control schemes, like model predictive control and fault tolerant control, are the issues addressed recently in AHS [6-11]. Most of such works [6, 7, 8, and 9] utilizes Unscented Kalman Filter (UKF) algorithm for estimating

$\dagger \quad$ Corresponding Author: Dept. of Electrical Engg., National Institute of Technology Calicut, Kerala, India. (meshij4u@gmail.com)

* Dept. of Electrical Engg., National Institute of Technology Calicut, Kerala, India. (mvv@nitc.ac.in)

Received: September 3, 2014; Accepted: April 18, 2016 the states of hybrid systems. UKF algorithm was originally proposed by S. Julier and Uhlmann [12] to overcome certain limitations such as Jacobian calculations in Extended Kalman Filter (EKF) [13] originated from the linear kalman filter [14], for the state estimation of the nonlinear systems. Later, some modifications were made in the basic structure of UKF for obtaining more accurate and robust state estimation of some classes of nonlinear system. Square Root UKF [15], Additive UKF, Rao-Blackwellised UKF [16] and iterated UKF [17] are some of those extended versions of UKF. These state estimation methods were also used in estimating the unknown process noise covariance [18] and discrete state variables [19]. The nonlinear model predictive control (NMPC) in [20] and fault tolerant model predictive control in [21], for hybrid systems, utilized this in its state estimation part. UKF based inverse dynamics controller was developed in [22] for controlling the non-measurable states of the hybrid systems. During the performance analysis in [22], it is found that the performance can be improved with the reduced estimation time requirement for the UKF algorithm and with the improved robustness under real time uncertainties. Authors have proposed methods to overcome these implementation issues [23] by designing EKF based and artificial neural network (ANN) based state estimators for estimating the states. Estimation time requirement can be very much reduced using EKF method, while robustness issue can be better handled by ANN based state estimator. Because of the analytical and statistical linearization involved in EKF and UKF algorithms respectively, robustness of these estimators is found to be poor especially under high degree of parameter 
uncertainties. It is shown in [23], because of the inherent self adaptation capability of ANN, ANN based state estimator gives better performance under a variety of operating conditions and uncertainties.

First part of this paper is focusing on review of different state estimation schemes, commonly employed for the state estimation of nonlinear hybrid systems, with an objective of proposing different controllers in improving the performance of such hybrid systems. As a first step in this direction, the effect of the nonlinear controller such as inverse dynamics controller is carried out on a bench mark model (hybrid three-tank system) and a comparative evaluation of the working of the control systems with this type of nonlinear controller acting on systems with three different estimation procedures namely EKF, UKF and ANNSE, is carried out. The advantages and disadvantages of these three closed loop control schemes with the estimation methods forming the elements of such closed loop control systems, are investigated in this work with experimentations. The investigation also includes detailed performance comparison of EKFC and ANNC with existing UKFC [22] when the plant is operating under various uncertainties and operating conditions such as servo/ regulatory operations, parameter uncertainties, initial condition mismatch, system faults etc.

The paper is giving the brief description of the benchmark hybrid three-tank system in section 2 along with the generic model developed in [9]. EKF [13], UKF [12] and author's ANN based state estimation algorithms [23] are briefly reviewed in section 3. IDC scheme with conventional measurement of output are extended to Estimator Based IDC and is presented in section 4. Experimental investigation of the system with different control mechanisms and comparison of performance are presented in Section 5. Finally, section 6 concludes this work and discusses scope for further studies in the state estimation and control of AHS. Process description of hybrid three-tank system is briefed in next section.

\section{Process Description $[9,24]$}

In the hybrid three-tank system, three tanks of uniform area $\left(A_{1}, A_{2}\right.$ and $\left.A_{3}\right)$ are connected to each other and to sump through seven hand valves and two variable speed pumps as in Fig. 1. The seven valve discharge coefficients are represented by $k_{1}$ to $k_{7}$. Once the positions of hand valves are fixed for the experiment, the corresponding discharge coefficients of each hand valves are found out by conducting simple experiments on the plant, which are given in table 1 . After computing $\mathrm{k}_{\mathrm{i}}$ (where, $\mathrm{i}=1,2, \ldots, 7$ ), the system is modeled using volume balance equations as in (1) - (3), where, $Q_{i}$ represent the flows through $\mathrm{i}^{\text {th }}$ hand valve and its equations are provided in (4) - (10).

The system is modeled as hybrid system, specifically AHS, as in [9] because of the interaction between continuous and discrete state variables. The flow through the middle inter connecting pipes can be better modeled with the help of discrete variables, $z_{1}$ and $z_{2}$ (defined in (11) and (12)).

With reference to Fig. 1

$$
\begin{gathered}
\frac{d h_{1}}{d t}=\frac{1}{\mathrm{~A}_{1}}\left[F_{i n_{1}}-Q_{1}-Q_{3}-Q_{5}\right] \\
\frac{d h_{2}}{d t}=\frac{1}{\mathrm{~A}_{2}}\left[F i_{n_{2}}-Q_{2}-Q_{4}-Q_{7}\right] \\
\frac{d h_{3}}{d t}=\frac{1}{\mathrm{~A}_{3}}\left[Q_{1}+Q_{2}+Q_{3}+Q_{4}-Q_{6}\right] \\
Q_{1}=\mathrm{k}_{1} \operatorname{sign}\left(h_{1}-h_{3}\right) \sqrt{2 \mathrm{~g}\left|h_{1}-h_{3}\right|} \\
Q_{2}=\mathrm{k}_{2} \operatorname{sign}\left(h_{2}-h_{3}\right) \sqrt{2 \mathrm{~g}\left|h_{2}-h_{3}\right|} \\
Q_{3}=z_{1} \mathrm{k}_{3} \sqrt{2 \mathrm{~g}\left|a\left(h_{1}-\mathrm{h}_{0}\right)-b\left(h_{3}-\mathrm{h}_{0}\right)\right|} \\
Q_{4}=z_{2} \mathrm{k}_{4} \sqrt{2 \mathrm{~g}\left|c\left(h_{2}-\mathrm{h}_{0}\right)-b\left(h_{3}-\mathrm{h}_{0}\right)\right|} \\
Q_{5}=\mathrm{k}_{5} \sqrt{2 \mathrm{~g}\left(h_{1}+\mathrm{h}_{\mathrm{d}_{1}}\right)} \\
Q_{6}=\mathrm{k}_{6} \sqrt{2 \mathrm{~g}\left(h_{3}+\mathrm{h}_{\mathrm{z}}\right)} \\
Q_{7}=\mathrm{k}_{7} \sqrt{2 \mathrm{~g}\left(h_{2}+\mathrm{h}_{\mathrm{d}_{2}}\right)}
\end{gathered}
$$

Depending on the flows, $Q_{3}$ and $Q_{4}$, discrete state variables, $z_{l}$ and $z_{2}$ may take the values $0,+1$ or -1 as given below

$$
z_{1}=\left\{\begin{aligned}
-1 ; & \text { if } Q_{3} \text { is a way from Tank } \\
0 ; & \text { if } Q_{3}=0 \\
+1 ; & \text { if } Q_{3} \text { is towards Tank }
\end{aligned}\right.
$$

Similarly

$$
z_{2}=\left\{\begin{aligned}
-1 ; & \text { if } Q_{4} \text { is a way from Tank } \\
0 ; & \text { if } Q_{4}=0 \\
+1 ; & \text { if } Q_{4} \text { is towards Tank }
\end{aligned}\right.
$$

As $Q 3$ and $Q 4$ are determined by the three levels, it is obvious that the discrete mode switching depends on the continuous state variables; hence the system comes under the definition of AHS [3]. The variables, $a, b$ and $c$ in (6) and (7) are temporary variables to indicate the levels in three tanks as below or above threshold level. The threshold level, $h_{0}$ is the height of middle interconnecting pipes and the tank bottom as in Fig. 1. The height difference between the $5^{\text {th }}, 6^{\text {th }}$ and $7^{\text {th }}$ hand valves from the bottom of the corresponding tanks are given by $h_{d 1}, h_{z}$ and $h_{d 2}$ respectively. The pumps used are variable speed pumps whose outflow ranges from 0 to rated value based on the input signal $(0-5 \mathrm{~V})$. The experimental setup specifications are given in Table 2 . 


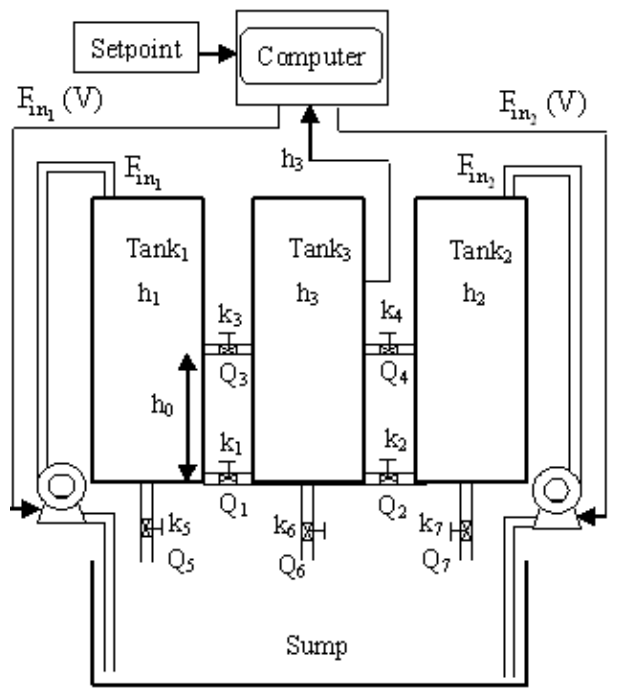

Fig. 1. Schematic diagram of hybrid three-tank system

Table 1. Discharge coefficients of hand valve

\begin{tabular}{c|c|c|c}
\hline $\begin{array}{c}\text { Discharge } \\
\text { coefficient }\end{array}$ & Value $\left(\mathrm{m}^{2}\right)$ & $\begin{array}{c}\text { Discharge } \\
\text { coefficient }\end{array}$ & Value $\left(\mathrm{m}^{2}\right)$ \\
\hline $\mathrm{k}_{1}$ & $2.6363 \times 10^{-5}$ & $\mathrm{k}_{4}$ & $3.4316 \times 10^{-5}$ \\
\hline $\mathrm{k}_{2}$ & $2.4891 \times 10^{-5}$ & $\mathrm{k}_{6}$ & $2.2538 \times 10^{-5}$ \\
\hline $\mathrm{k}_{3}$ & $3.7984 \times 10^{-5}$ & $\mathrm{k}_{5}, \mathrm{k}_{7}$ & 0 \\
\hline
\end{tabular}

Table 2. Different system parameters and variables

\begin{tabular}{c|c|c|c}
\hline $\begin{array}{c}\text { Variables and } \\
\text { Parameter }\end{array}$ & Value & $\begin{array}{c}\text { Variables and } \\
\text { Parameter }\end{array}$ & Value \\
\hline Tank height & $0.60 \mathrm{~m}$ & Input voltage to pump & $0-5 \mathrm{~V}$ \\
\hline Over flow height & $0.55 \mathrm{~m}$ & Tank inner diameter & $0.15 \mathrm{~m}$ \\
\hline $\begin{array}{c}\text { Rated flow of } \\
\text { pumps }\end{array}$ & $240 \mathrm{lph}$ & $\begin{array}{c}\text { Diameter of } \\
\text { Connecting pipes }\end{array}$ & $0.0125 \mathrm{~m}$ \\
\hline
\end{tabular}

This nonlinear model is used as the prediction model of the state estimation algorithm and the EKF and UKF algorithms are briefed in the next section.

\section{State Estimation Algorithms}

Out of different state estimation schemes, UKF based scheme is the mostly suggested technique for hybrid systems as it is suitable for highly nonlinear systems $[8,9$, 20-22]. The nonlinear and derivative free nature [21] of this algorithm makes the efficient state estimation of highly complex and nonlinear systems like hybrid systems possible. But, EKF algorithm uses local linearization by Taylor series expansion, which requires the calculation of Jacobians of state transition operator at each time step. So this method is supposed to be complex for higher order systems [21]. Because of the discontinuity in hybrid systems due to the switching of discrete variables, it was suggested in [21], not to use EKF for the state estimation of AHS, especially in the regions where the discrete variables undergo frequent variations. If this problem with EKF can be solved effectively, it will be a good choice for

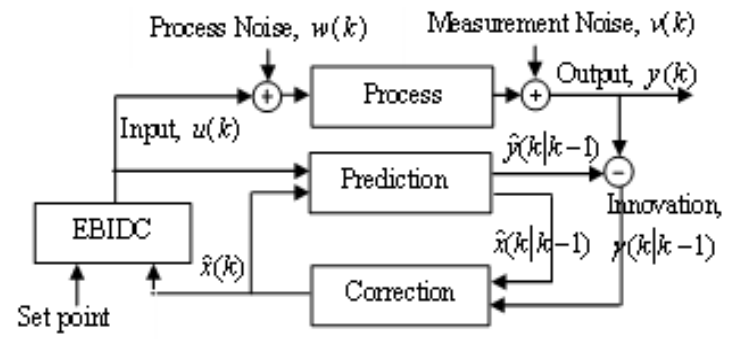

Fig. 2. General block diagram of estimator based controller

the state estimation of faster dynamics AHS [23] such as thermostats.

As per [21], the usage of EKF is not preferred at the point of mode switching. In the current problem, this condition occurs only when the level in tank is exactly 0.3 $\mathrm{m}$. But the chance for reaching this situation is negligibly small as the Jacobian calculation is carried out only once in a sampling time. EKF algorithm for the AHS is developed by defining different Jacobian functions for each mode of operation based on the discrete variables $\left(z_{1}\right.$ and $\left.z_{2}\right)$. Also if the unexpected situation (where level is exactly $0.3 \mathrm{~m}$ ) occurs, then the Jacobian of the previous sampling instant is taken for the correction part of the state estimator. This may lead to negligible biased estimate at that particular sampling instant. The EKF, UKF and ANN based state estimation algorithms are provided in the following subsections. The general block diagram of the estimator along with plant and controller is provided in Fig. 2 [22]. Different notations used in this section are similar to that in [20]

Dimension and description of variables used in the algorithms are given below.

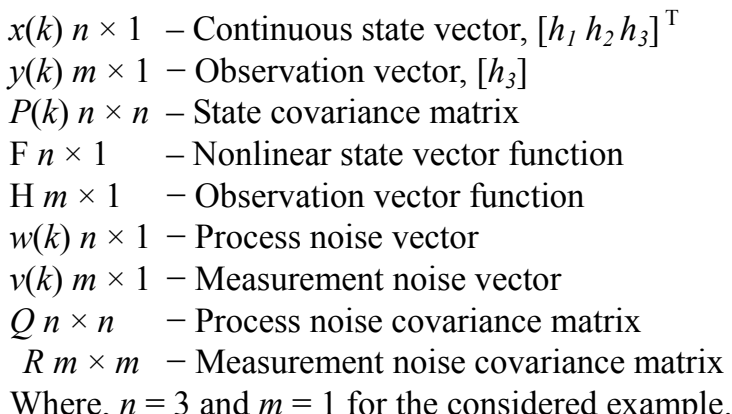

\subsection{EKF- based state estimation algorithm [13]}

The EKF algorithm, which is the nonlinear extension to the linear Kalman Filter [14], was developed for the state estimation of nonlinear systems. A prediction - correction structure is used for the state estimation as in Fig. 2. For the implementation of EKF algorithm (also for UKF), the knowledge about the process dynamics, the statistical characteristics of the system and the measurement devices should be available. The EKF algorithm for the state estimation, summarized below is taken from [13]. 
1. Initial values for the estimates of state, $\hat{x}(k-1)$ and state covariance, $\hat{P}(k-1)$ has to be assumed properly by utilizing the knowledge about the system.

2. Measure the input and output variables $(u(k)$ and $y(k))$ from the process and feed the inputs to the prediction model to get apriori estimates of the states,

$$
\hat{x}(k \mid k-1)=\mathrm{F}(\hat{x}(k-1), u(k))
$$

Where, $\mathrm{F}$ is the nonlinear state function described by (1) - (3)

3. Calculate apriori estimates of the state covariance as

$$
\hat{P}(k \mid k-1)=\Phi(k) \hat{P}(k-1) \Phi^{\mathrm{T}}(k)+Q
$$

Where, $\Phi(k)$ is the state Jacobian matrix or partial derivative of the nonlinear state function, $\mathrm{F}$ with respect to $x$ at $x(k-1)$ and is given below. The process noise covariance, $Q$ is used as the tuning factor for the estimator for obtaining the optimum estimates.

$$
\Phi(k)=[\partial \mathrm{F} / \partial x][\hat{x}(k-1)]
$$

4. The output prediction from the model is given below.

$$
\hat{y}(k \mid k-1)=\mathrm{H}[\hat{x}(k \mid k-1)]
$$

Where, $\mathrm{H}($.$) is the nonlinear output function.$

5. The innovation between the actual measured output from the plant and the measurement prediction is generated as follows

$$
\gamma(k \mid k-1)=y(k)-\hat{y}(k \mid k-1)
$$

6. Covariance matrix of the innovation is computed as follows

$$
\mathrm{V}(k)=\mathrm{C}(k) \hat{P}(k \mid k-1) \mathrm{C}^{\mathrm{T}}(k)+R
$$

Where, $R$ is the measurement covariance and $\mathrm{C}(\mathrm{k})$ is the output Jacobian matrix calculated as follows.

$$
\mathrm{C}(k)=[\partial \mathrm{H} / \partial x]_{[\hat{x}(k-1)]}
$$

7. The Kalman gain has to be calculated as below for the correction of apriori estimates to get aposteriori estimates,

$$
K(k)=\hat{P}(k \mid k-1) \mathrm{C}^{\mathrm{T}}(k) \mathrm{V}^{-1}(k)
$$

8. Finally, using kalman gain and innovation, state variable and state covariance are corrected as follows.

$$
\begin{gathered}
\hat{x}(k)=\hat{x}(k \mid k-1)+K(k) \gamma(k \mid k-1) \\
\hat{P}(k)=[\mathrm{I}-K(k) \mathrm{C}(k)] \hat{P}(k \mid k-1)
\end{gathered}
$$

These new values of state variables and covariance estimates are given as the input to the Eqs. (13) and (14) for the next iteration and so on. The main disadvantage of this algorithm is that it requires the calculation of Jacobians at every sampling instant [13].

\subsection{UKF-based state estimation algorithm [12]}

UKF algorithm is mostly used state estimation scheme for the state estimation of hybrid systems, because it was proposed for highly nonlinear systems and it does not involve the Jacobian matrix calculation in each time step. It uses statistical methods for the linearization around the operating point. For that, sigma points calculation around current mean and covariance of state, are carried out at the starting of each iteration and these sigma points are fed to the prediction model to obtain time updated new sigma points. Steps for developing computational algorithm for state estimation using UKF approach, which is taken from [12] are listed below.

1. Assume the proper initial values for state and covariance as in EKF algorithm

2. Measure the input and output from the process

3. Generate sigma points as

$$
\begin{gathered}
\hat{x}^{(0)}=\hat{x}(k-1) \\
\hat{x}^{(i)}=\hat{x}(k-1) \pm \sqrt{(n+\lambda) \hat{P}(k-1)} \\
\text { Here, } \quad \lambda=\alpha^{2}(n+\kappa)-n
\end{gathered}
$$

The value of $i$ may vary from 1 to $2 n$. Where, $n$ is the length of state vector. The spread of sigma points $\alpha$ can be used as another tuning factor for the estimator, whose value lies between 0.001 and 1 . The optimum value of secondary scaling factor $\kappa$ is 0 for state estimation problems [15].

4. By passing these $2 n+1$ sigma points through the prediction model, time updated new sigma points for states and output are obtained.

$$
\begin{gathered}
\hat{x}^{(i)}(k \mid k-1)=\mathrm{F}\left(\hat{x}^{(i)}(k-1), u(k)\right) \\
\hat{y}^{(i)}(k \mid k-1)=\mathrm{H}\left(\hat{x}^{(i)}(k \mid k-1)\right)
\end{gathered}
$$

5. Based on their statistics apriori estimates of state, $\hat{x}(k \mid k-1)$ and covariance, $\hat{P}(k \mid k-1)$ are calculated.

$$
\begin{gathered}
\hat{x}(k \mid k-1)=\sum_{i=0}^{2 n} W_{m}^{(i)} \hat{x}^{(i)}(k \mid k-1) \\
\hat{P}(k \mid k-1)=\sum_{i=0}^{2 n} W_{c}^{(i)}\left\{\left[\tilde{x}^{(i)}\right]\left[\tilde{x}^{(i)}\right]^{\mathrm{T}}\right\}+Q
\end{gathered}
$$

where, 


$$
\tilde{x}^{(i)}=\hat{x}^{(i)}(k \mid k-1)-\hat{x}(k \mid k-1)
$$

6. Apriori estimate of output prediction is computed as

$$
\hat{y}(k \mid k-1)=\sum_{i=0}^{2 n} W_{m}^{(i)} \hat{y}^{(i)}(k \mid k-1)
$$

The weights involved in computing the apriori estimates (28-31) are obtained as below.

$$
\begin{gathered}
W_{m}^{(0)}=\lambda /(n+\lambda) \\
W_{c}^{(0)}=\lambda /(n+\lambda)+\left(1-\alpha^{2}+\beta\right) \\
W_{m}^{(i)}=W_{c}^{(i)}=1 / 2(n+\lambda)
\end{gathered}
$$

In (33), $\beta$ is employed for incorporating the knowledge about the state distribution. In this case, the distribution is considered as Gaussian and the value of $\beta$ for Gaussian distribution is 2 [15].

7. Auto covariance of the innovation and cross covariance between the innovation and apriori estimates are given below, which were used for the calculation of Kalman gain.

$$
P_{y y}=\sum_{i=0}^{2 n} W_{c}^{(i)}\left[\tilde{y}^{(i)}\right]\left[\tilde{y}^{(i)}\right]^{\mathrm{T}}+R
$$

$$
P_{x y}=\sum_{i=0}^{2 n} W_{c}^{(i)}\left[\tilde{x}^{(i)}\right]\left[\tilde{y}^{(i)}\right]^{\mathrm{T}}
$$

where,

$$
\tilde{y}^{(i)}=\hat{y}^{(i)}(k \mid k-1)-\hat{y}(k \mid k-1)
$$

8. Calculation of kalman gain using $P_{y y}$ and $P_{x y}$ is carried out.

$$
K(k)=P_{x y} P_{y y}^{-1}
$$

9. Correction of apriori estimates of states and covariance using kalman gain, are done as follows.

$$
\begin{gathered}
\hat{x}(k)=\hat{x}(k \mid k-1)+K(k)[y(k)-\hat{y}(k \mid k-1)] \\
\hat{P}(k)=\hat{P}(k \mid k-1)-K(k) P_{y y} K^{\mathrm{T}}(k)
\end{gathered}
$$

The steps 2 to 9 are repeated at all the iterations of the entire process run.

\subsection{ANN- based state estimation algorithm [23]}

It is developed for eliminating the linearization of the nonlinear state function involved in the correction part of EKF and UKF algorithm. In this scheme, the correction

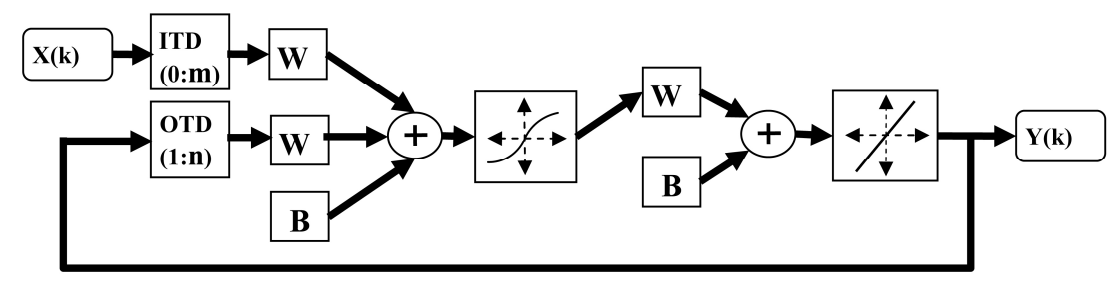

(a)

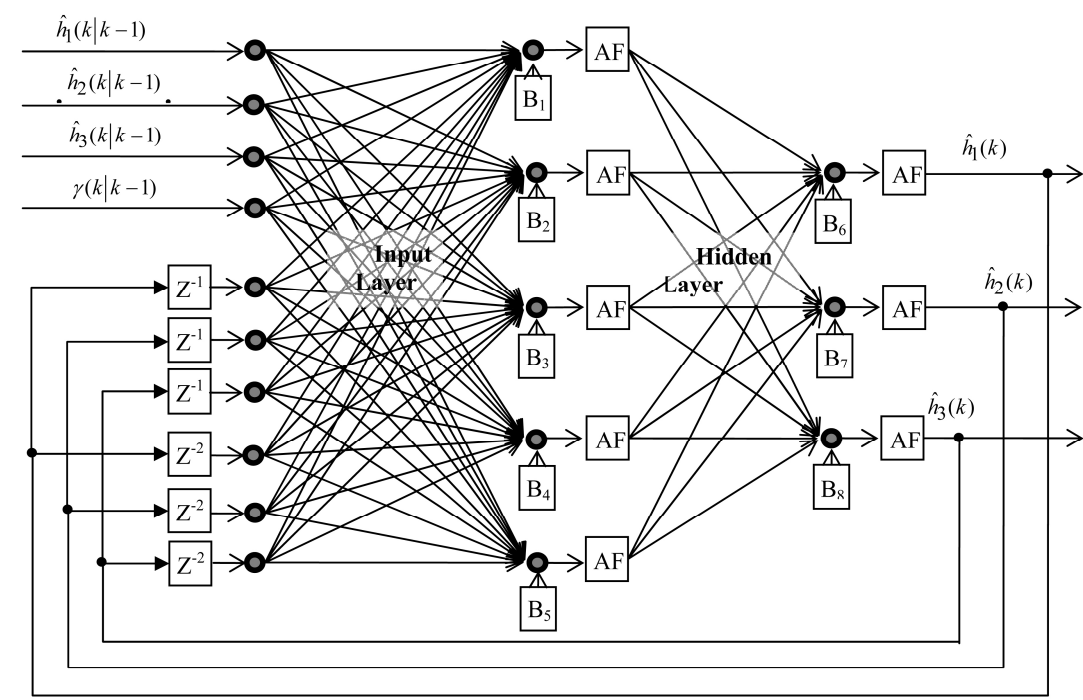

(b)

Fig. 3. General NARX structured ANN, (b) NARX structure for the considered example 
part is replaced by a nonlinear ANN model. Since ANN model is a mathematical abstraction using the empirical models, this method can be a better choice for replacing the correction part of the estimation scheme. Because of the huge data (covering the entire operating region) based training of ANN, this method provides more robust estimation of states. The ANN is a black box modeling tool in which the linear combination of inputs along with activation function (AF) gives the output. To capture the dynamic behavior of the correction part in a better way, a dynamic neural network (NARX) is suggested.

NARX, the recurrent dynamic neural network is having feedback connections from different layers of the network. The current output can be predicted as a function of present and past inputs and past outputs as given below, in which $\mathrm{Y}$ and $\mathrm{X}$ represent the outputs and inputs of the network respectively and $\mathrm{K}_{\mathrm{NN}}$ is a nonlinear ANN function.

$$
\mathrm{Y}(\mathrm{k})=\mathrm{K}_{\mathrm{NN}}\{\mathrm{X}(\mathrm{k}), \ldots, \mathrm{X}(\mathrm{k}-\mathrm{m}), \mathrm{Y}(\mathrm{k}-1), \ldots, \mathrm{Y}(\mathrm{k}-\mathrm{n})\}
$$

A sequence of current and past input vectors $(\mathrm{X}(\mathrm{k})$, $\mathrm{X}(\mathrm{k}-1), \ldots, \quad \mathrm{X}(\mathrm{k}-\mathrm{m}))$ are obtained by passing $\mathrm{X}(\mathrm{k})$ through an input time delay unit, ITD $(0: m)$. Similarly output time delay unit, $\operatorname{OTD}(1: \mathrm{n})$ provides a sequence of past output vectors $(\mathrm{Y}(\mathrm{k}-1), \ldots, \mathrm{Y}(\mathrm{k}-\mathrm{n}))$. For the considered problem, the input to the neural network,

$\mathrm{X}(k)=\left[\hat{h}_{1}(k \mid k-1), \hat{h}_{2}(k \mid k-1), \hat{h}_{3}(k \mid k-1), \gamma(k \mid k-1)\right]^{\mathrm{T}}$ and the output, $\mathrm{Y}(k)=\left[\hat{h}_{1}(k), \hat{h}_{2}(k), \hat{h}_{3}(k)\right]^{\mathrm{T}}$.

Training of the ANN is done using the data obtained from the EKF based state estimation. Tuning of the ANN is done by varying Hidden layer neurons, number of output delays (n) and number of input delays (m) by keeping the integral square error (ISE) as the performance measure. After training and tuning of NARX the weights (W) and biases (B) are obtained, by making use of MATLAB functions. The general NARX diagram and the detailed structure for the considered problem are given in Fig. 3. The parameters related to the NARX are listed in Table 3. Now the correction part of state estimation scheme (Fig. 2) can be replaced with this ANN

The steps involved in ANN based state estimation

Table 3. Values of different ANN parameters

\begin{tabular}{c|c}
\hline Parameter & Value \\
\hline ANN Structure & NARX \\
\hline No. of hidden layers & 1 \\
\hline Hidden Layer neurons & 5 \\
\hline Hidden layer activation function & 'tansigmoid' \\
\hline Output layer activation function & 'purelin' \\
\hline No of exogenous inputs & 0 \\
\hline No. of delayed inputs & 3 \\
\hline No of outputs & 2 \\
\hline No. of feedback output delays & Back propagation \\
\hline Training method & Levenberg Marquardt \\
\hline Training function & Mean Square Error
\end{tabular}

algorithm are summarized below.

1. Assume the proper values for the initial state vector.

2. Measure the input and output variables from the plant.

3. Present the input and assumed state vector to the prediction model to obtain apriori state estimates as below

$$
\hat{x}(k \mid k-1)=\mathrm{F}(\hat{x}(k-1), u(k))
$$

4. Find the apriori output estimates as

$$
\hat{y}(k \mid k-1)=\mathrm{H}[\hat{x}(k \mid k-1)]
$$

5. Calculate the innovation between the plant output and apriori output estimates as

$$
\gamma(k \mid k-1)=y(k)-\hat{y}(k \mid k-1)
$$

6. Equation 41 can be re written to obtain aposteriori estimates of the state as follows.

$$
\hat{x}(k)=\mathrm{K}_{\mathrm{NN}}\{\operatorname{ITD}(\hat{x}(k \mid k-1), \gamma(k \mid k-1)), \mathrm{OTD}(\hat{x}(k))\}
$$

Repeat the steps 2 to 6 , at each sampling instants of the entire period of operation, with recent state estimates as input to the model. The estimated values of state using different state estimators are fed to the inverse dynamics controller for controlling the non-measurable state variables and the control law required to realize this which is reported in [22] is reproduced in the next section.

\section{Estimator Based Controller [22]}

If the process is of nonlinear type, the conventional controllers $(\mathrm{P}, \mathrm{P}+\mathrm{I}$ or $\mathrm{P}+\mathrm{I}+\mathrm{D})$ will not give satisfactory performance in all its operating regions. Some kind of nonlinear controllers have to be selected for such control applications. In hybrid systems, for the controller design, its mode switching property has also to be taken in to account. For such processes, model based control schemes are proposed in the literature [7-9, 20-22] for obtaining the satisfactory control of the output variables.

In this work, as in the case of UKFC in [22], an estimator based inverse dynamics controller (EBIDC) is implemented for controlling the non-measurable states of AHS. Its general block diagram is given in Fig. 2. These non- measurable variables are estimated using different state estimation algorithms explained in section 3. These estimated states are fed to the EBIDC based on which the corrective action is taken. In the benchmark hybrid threetank system, out of three state variables $\left(h_{1}, h_{2}\right.$ and $\left.h_{3}\right), h_{3}$ is the only sensed output state. The control problem is identified as to control the levels $h_{l}$ and $h_{2}$ using the output 
measurement alone. Because of the reduction in the number of sensors, the EBIDC can be termed as reduced sensor control scheme.

The control law derived in [22] is given below.

$$
\begin{aligned}
& F_{i n_{1}}=\mathrm{A}_{1} \mathrm{C}_{1}\left(\mathrm{~h}_{1 \mathrm{sp}}-\hat{h}_{1}\right)+\hat{Q}_{1}+\hat{Q}_{3}+\hat{Q}_{5} \\
& F_{i n_{2}}=\mathrm{A}_{2} \mathrm{C}_{2}\left(\mathrm{~h}_{2 \mathrm{sp}}-\hat{h}_{2}\right)+\hat{Q}_{2}+\hat{Q}_{4}+\hat{Q}_{7}
\end{aligned}
$$

Where, $\mathrm{C}_{1}$ and $\mathrm{C}_{2}$ are controller tuning parameter, and its values are varied from 0 to 1 on separate runs and the integral square error between the controlled variable and the set point, is noted. The values of $\mathrm{C}_{1}$ and $\mathrm{C}_{2}$, which give the minimum ISE, are selected as the tuning parameter. The $h_{1 s p}$ and $h_{2 s p}$ are the corresponding externally fed desired values of water levels that are to be controlled. The estimated values of corresponding levels are represented by $\hat{h}_{l}$ and $\hat{h}_{2}$ respectively.

\section{Experimental Results}

Different EBIDC algorithm were implemented separately on the experimental setup shown in Fig. 4. In addition to the experimental setup, other tools used for the real time implementation are the software LabVIEW and the NI DAQ pad (USB6251). USB 6251 has 16 analog channels for data acquisition. After determining the discharge coefficients of different hand valves, Model developed in section II is validated using this experimental setup and then the state estimation algorithms are validated [23].

Once the performances of the estimators are satisfied [23], these estimators are used in the EBIDC, to control the non-measurable states of the experimental setup of AHS. In the real system, the performance experimentally obtained by using EKFC, UKFC and ANNC, is evaluated in detail under a variety of operating conditions, constraints, uncertainties and system faults, so that the robustness of the proposed aproaches (EKFC and UKFC) for AHS can be well studied. Both qualitative and quantitative

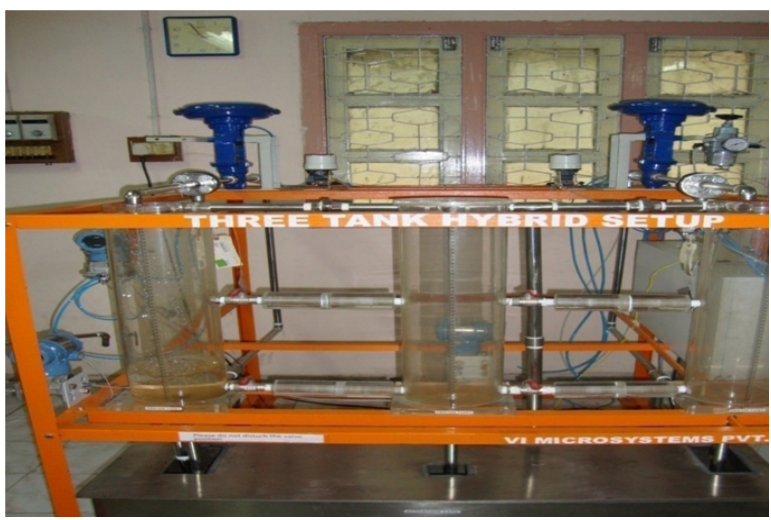

Fig. 4 Experimental set up of hybrid three-tank system comparisons were carried under various situation of the plant operation, which are presented in the following subsections.

\subsection{Servo and regulatory operation}

Set point tracking capability of the closed loop system is studied by introducing a step change in setpoint of magnitude $0.04 \mathrm{~m}$ at $100^{\text {th }}$ sampling instant and the qualitative comparison of the performance is given in Fig. 5(a). In this case ISE, set point tracking time, control signal generation time etc. were considered as the quantitative performance measures, and they are tabulated in Table 4. From this it is clear that even though all the controllers respond to the set point variations very well, EKFC is giving slightly better performance while considering the ISE criteria and control signal generation time.

For evaluating the regulatory operation of the system,

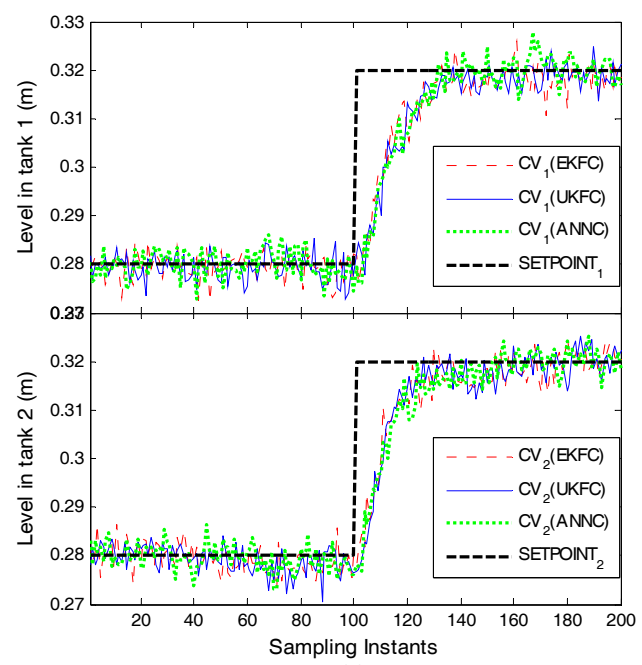

(a)

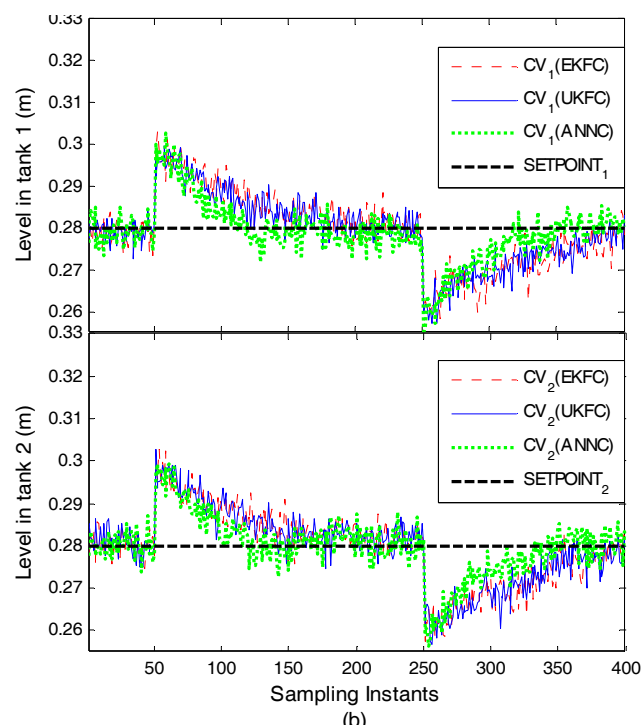

(b)

Fig. 5 Controlled Levels $\left(h_{l}\right.$ and $\left.h_{2}\right)$ in hybrid three-tank system: (a) servo response; (b) regulatory response 
Table 4. Performance measure (servo operation)

\begin{tabular}{c|c|c|c}
\hline Controller & $\mathrm{ISE}_{1,}\left(\mathrm{~m}^{2} \mathrm{~s}\right)$ & $\mathrm{ISE}_{2},\left(\mathrm{~m}^{2} \mathrm{~s}\right)$ & $\begin{array}{c}\text { Setpoint Tracking } \\
\text { Time, }(\mathrm{s})\end{array}$ \\
\hline EKFC & 0.0149 & 0.0132 & 34 \\
\hline UKFC & 0.0160 & 0.0148 & 36 \\
\hline ANNC & 0.0172 & 0.0144 & 32 \\
\hline
\end{tabular}

Table 5. Performance measure (regulatory operation)

\begin{tabular}{c|c|c|c|c}
\hline Controller & $\begin{array}{c}\mathrm{ISE}_{1}, \\
\left(\mathrm{~m}^{2} \mathrm{~s}\right)\end{array}$ & $\begin{array}{c}\mathrm{ISE}_{2}, \\
\left(\mathrm{~m}^{2} \mathrm{~s}\right)\end{array}$ & $\begin{array}{c}\text { Disturbance } \\
\text { Rejection } \\
\text { Time, }(\mathrm{s})\end{array}$ & $\begin{array}{c}\text { Control Signal } \\
\text { Generation } \\
\text { time, }(\mathrm{s})\end{array}$ \\
\hline EKFC & 0.0308 & 0.0296 & 130 & 0.0328 \\
\hline UKFC & 0.0277 & 0.0304 & 120 & 0.1849 \\
\hline ANNC & 0.0200 & 0.0193 & 70 & 0.1038 \\
\hline
\end{tabular}

Table 6. Performance measure (Initial state vector mismatch)

\begin{tabular}{c|c|c|c|c}
\hline Controller & $\begin{array}{c}\mathrm{ISE}_{1}, \\
\left(\mathrm{~m}^{2} \mathrm{~s}\right)\end{array}$ & $\begin{array}{c}\mathrm{ISE}_{2}, \\
\left(\mathrm{~m}^{2} \mathrm{~s}\right)\end{array}$ & $\begin{array}{c}\text { Setpoint } \\
\text { Tracking Time, } \\
(\mathrm{s})\end{array}$ & $\begin{array}{c}\text { Control Signal } \\
\text { Generation } \\
\text { time, }(\mathrm{s})\end{array}$ \\
\hline EKFC & 0.3151 & 0.5480 & 275 & 0.0293 \\
\hline UKFC & 0.1767 & 0.3175 & 180 & 0.1935 \\
\hline ANNC & 0.0214 & 0.0592 & 68 & 0.1017 \\
\hline
\end{tabular}

disturbances are introduced at $50^{\text {th }}$ and $250^{\text {th }}$ sampling instants by directly adding and removing some quantity of water to or from the tanks. In this case, disturbance rejection time is also considered as performance index (Table 5). Graphical comparison of the result, provided in Fig. 5(b), shows that, the disturbance rejection capability of the controllers are found to be good and, ANNC is quickly rejecting the effects of disturbance when compared EKFC and UKFC and is quantitatively compared in Table 5.

\subsection{Initial state vector mismatch}

The first step of implementing all these methods (EKFC, UKFC and ANNC) is the selection of initial state vector for the model. This assumed value need not be equal to the actual levels of the process as sensors for some levels $\left(h_{1}\right.$ and $h_{2}$ ) are assumed to be unavailable. So it is necessary to evaluate the performance of the controllers under initial condition mismatch. Initial state vector considered for the model is [ [ $\left.\begin{array}{lll}0 & 0 & 0.23\end{array}\right]$ and that for the plant is [ $\left.\begin{array}{llll}0.28 & 0.28 & 0.23\end{array}\right]$. So a mismatch of $0.28 \mathrm{~m}$ is considered in the initial values of $h_{1}$ and $h_{2}$.and the performance is given in Fig. 6 and the quantitative comparison is given in Table 6. Along with general performance measures setpoint tracking time is also considered as an additional performance measure. ANNC is giving the best performance compared to that of others which can be observed from Table 6 and Fig. 6. Also the maximum deviation from set point due to this initial condition mismatch is lowest for ANNC scheme.

\subsection{Parameter uncertainties}

As both the state estimation and control algorithms are
Table 7. Performance measure (Parameter uncertainties)

\begin{tabular}{c|c|c|c|c|c}
\hline $\begin{array}{c}\text { Parameter } \\
\text { mismatch }\end{array}$ & Controller & $\begin{array}{c}\text { ISE }_{1} \\
\left(\mathrm{~m}^{2} \mathrm{~s}\right)\end{array}$ & $\begin{array}{c}\text { ISE }_{2} \\
\left(\mathrm{~m}^{2} \mathrm{~s}\right)\end{array}$ & $\begin{array}{c}\text { Control Signal } \\
\text { Generation time }(\mathrm{s})\end{array}$ & $\begin{array}{c}\text { Offset } \\
(\mathrm{m})\end{array}$ \\
\hline \multirow{3}{*}{$+10 \%$} & EKFC & 0.0128 & 0.0131 & 0.0294 & +0.0087 \\
\cline { 2 - 6 } & UKFC & 0.0124 & 0.0120 & 0.2112 & +0.0079 \\
\cline { 2 - 6 } & ANNC & 0.0045 & 0.0069 & 0.0999 & +0.0039 \\
\hline \multirow{3}{*}{$-10 \%$} & EKFC & 0.0352 & 0.0341 & 0.0373 & -0.0152 \\
\cline { 2 - 6 } & UKFC & 0.0297 & 0.0290 & 0.2010 & -0.0138 \\
\cline { 2 - 6 } & ANNC & 0.0122 & 0.0131 & 0.1037 & -0.0064 \\
\hline
\end{tabular}

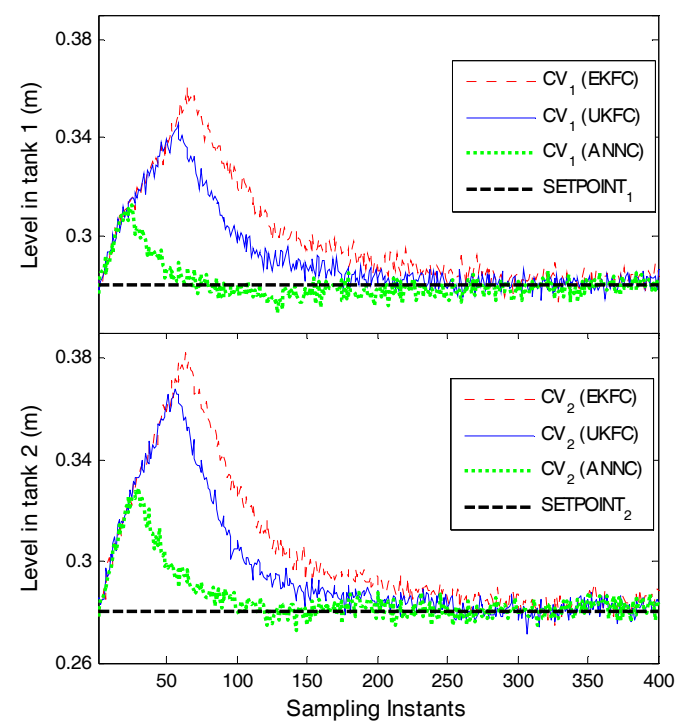

Fig. 6. Controlled Levels $\left(h_{l}\right.$ and $\left.h_{2}\right)$ in hybrid three-tank system under initial state vector mismatch

model dependant, the parameter used in the model may play an important role in the control action. The parameters, area of the tanks and valve coefficients are found by direct measurements, conducting experiments and doing steady state analysis. So there is a chance of measurement and calculation error due to human involvements. This condition is included in the studies by introducing a mismatch of $10 \%$ of actual value in area of all tanks and hand valve coefficients. Both positive and negative mismatch conditions are evaluated and the results are given in Fig. 7 and the performance measures are given in Table 7.

From the results presented in Fig. 7 and Table 7, it is clear that ANNC outperforms its counterparts under parameter uncertainties as it is giving lowest ISE. Also, the offset in the controlled variable is considered as a special performance index in this situation and from the results it is lesser in the case of ANNC when compared with that of EKFC and UKFC.

\subsection{Hand valve faults}

One of the main hand valve faults is leakage of water in the closed condition. Leakage fault is introduced in the permanently closed fifth hand valve, at $100^{\text {th }}$ sampling instants onwards in one run. In another run, clogging of 
Table 8. Performance measure (valve faults)

\begin{tabular}{c|c|c|c|c}
\hline Condition & Controller & $\begin{array}{c}\mathrm{ISE}_{1} \\
\left(\mathrm{~m}^{2} \mathrm{~s}\right)\end{array}$ & $\begin{array}{c}\mathrm{ISE}_{2} \\
\left(\mathrm{~m}^{2} \mathrm{~s}\right)\end{array}$ & $\begin{array}{c}\text { Control Signal } \\
\text { Generation time (s) }\end{array}$ \\
\hline \multirow{3}{*}{ Leakage } & EKFC & 0.1110 & 0.0072 & 0.0518 \\
\cline { 2 - 5 } & UKFC & 0.1026 & 0.0059 & 0.1883 \\
\cline { 2 - 5 } & ANNC & 0.0600 & 0.0077 & 0.0768 \\
\hline \multirow{3}{*}{ Clogging } & EKFC & 0.7638 & 0.7610 & 0.0268 \\
\cline { 2 - 5 } & UKFC & 0.2616 & 0.2613 & 0.1630 \\
\cline { 2 - 5 } & ANNC & 0.0082 & 0.0078 & 0.0943 \\
\hline
\end{tabular}

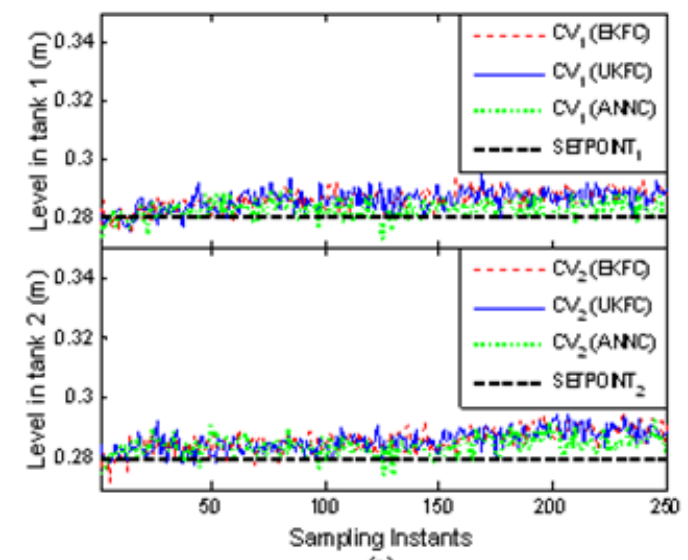

(a)

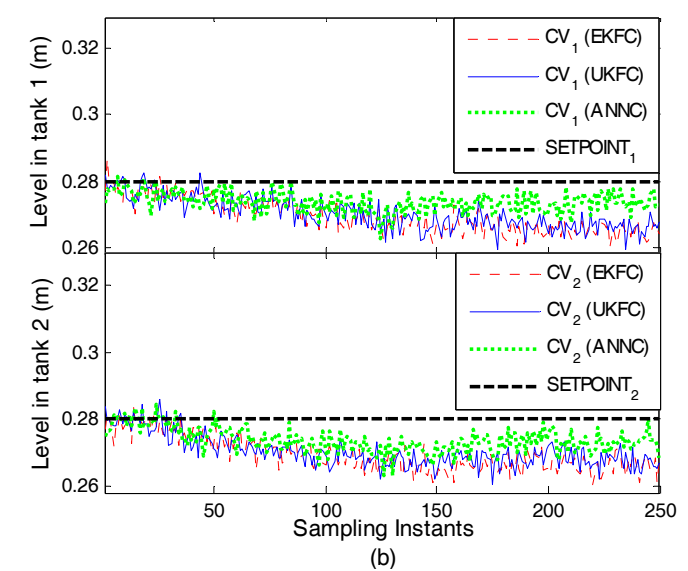

Fig. 7 Controlled Levels $\left(h_{1}\right.$ and $h_{2}$ ) in hybrid three-tank system Parameter mismatch (a) $+10 \%$ (b) $-10 \%$

hand valve, another type of fault, is introduced by partially reducing the flow through $6^{\text {th }}$ hand valve at $100^{\text {th }}$ sampling instant. How the controllers are responding to these faults is given in Fig. 8 and the performance measures is given in Table 8.

From Table 8, the ANNC is giving the lesser ISE values which confirm the better performance of ANNC under these hand valve faults.

In all the above mentioned cases of plant operation, even though the ISE is largest in the case of EKFC, the control signal generation time lowest for EKFC. Also highest control-signal generation time is obtained in the case of UKFC. So EKFC will be a better option for controlling the state variables of faster dynamic AHS.

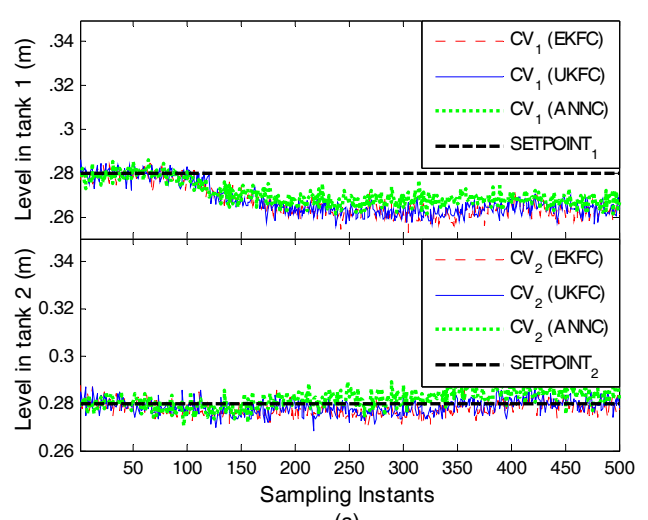

(a)

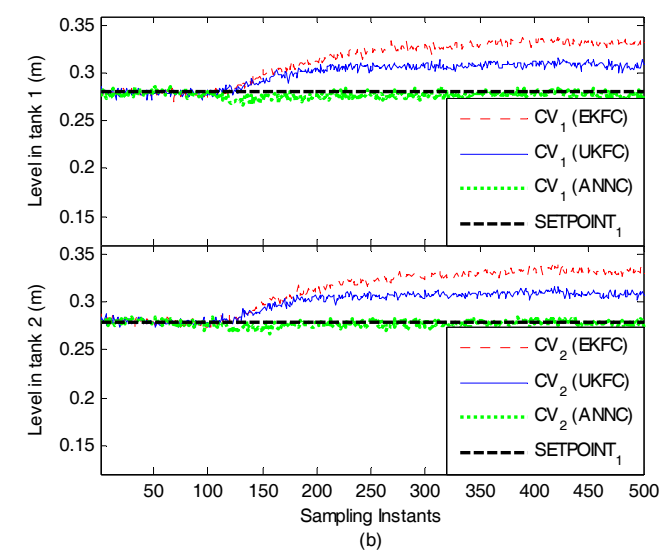

Fig. 8 Controlled Levels $\left(h_{1}\right.$ and $\left.h_{2}\right)$ in hybrid three-tank system under hand valve faults: (a) Leakage; (b) Clogging

\section{Conclusion}

Main Contributions of this paper are as follows.

- A robust ANNC and faster EKFC, for controlling the non-measurable states of autonomous hybrid systems, have been proposed.

- Performance evaluation was carried out on an experimental setup of hybrid three-tank system and their performance was compared with that of UKFC

- Using ANNC implemented for the considered system, a considerable improvement in the ISE in most of the situations are obtained as reported in section $\mathrm{V}$.

- Using the EKFC implementation, the control signal generation time was considerably reduced.

Quantitative analysis was made in terms of ISE, control signal generation time as general performance index and set point tracking time and disturbance rejection time in some specific situations. The robustness in controlling the non-measurable states, under some real time operating constraints, was observed by conducting experiments on the real plant and the results are reported in section 5. In all 
the cases mentioned in section 5, except in servo operation of the closed loop system, the ISE is the lowest for ANNC which implies that ANNC has more robustness when compared to EKFC and UKFC in controlling the nonmeasurable states of AHS under the above mentioned conditions. The robustness and accuracy of the system with ANNC are achieved due to the following reasons.

- In the correction part of EKFC and UKFC, some kinds of linearization of nonlinear state function around the operating point are involved. But, ANNC uses a nonlinear $\mathrm{ANN}$, which is a mathematical abstraction using empirical models trained using the data collected from the system, to correct the apriori estimates.

- The correction part of ANNC is completely parameter independent, which gives the better state estimates under parameter mismatch.

- ANN has built in noise rejection capability.

- Dynamic ANN structure (NARX) selected is suitable for this application.

- Training of the ANNC is carried out with a very huge set of data which covers the entire operating region of the process.

To conclude, it is highly recommended to use ANNC for controlling the non-measurable states of AHS especially when the robustness is the main concern of the control. Even though EKFC is giving, higher ISE values in all the cases, it is better to go for EKFC when the states of faster dynamics processes have to be controlled. The controlsignal generation time for EKFC is nearly $1 / 4^{\text {th }}$ that for ANNC and $1 / 8^{\text {th }}$ that for UKFC as reported in section V.

In conditions where parameter change occurs, an offset in the controlled variable is obtained. If the parameters are estimated and updated in the model frequently, the offset can be removed. Future works may be addressed in this direction. Also, the results obtained are motivating to derive new algorithms for the AHS under uncertainties

\section{Acknowledgement}

The authors greatly acknowledge the help rendered by Dr. J. Prakash, Professor and Head, Instrumentation Engineering Department, MIT Campus Anna University, Chennai, India by providing facilities to carry out the experimental work in Process control laboratory of his department

\section{References}

[1] N. Sriram., B. Gautam. "Model-based diagnosis of hybrid systems", IEEE trans. on systems, man and cybernetics, part a: systems and humans, vol. 37 issue. 3 , pp. 348-361, 2007
[2] Thomas. Krilavičius, "Hybrid Techniques for Hybrid Systems", PhD dissertation, University of Twente, Enschede, The Netherlands, Sep. 2006.

[3] W. P. M. H. Heemels, D. Lehmann, J. Lunze, B. De Schutter, "Introduction to hybrid systems", Cambridge university press, pp. 3-30, 2009.

[4] M. Sarailoo, Z. Rahmani, B. Rezaie, "Modeling of Three-Tank System with Nonlinear Valves Based on Hybrid System Approach", journal of control engg and technology, vol. 3, issue 1, pp. 20-23, 2013.

[5] Cyril J., V. I. George, P. R. Venkateswaran, "Discrete controller design for a hybrid three - tank system", Int. Journal on advanced science engineering information technology, Vol. 2, No. 1, pp. 40-44, 2012.

[6] Prakash Jagadeeshan., M. Elenchenzhiyan S.L. Shah, "State estimation of a non-linear hybrid system using an interacting multiple model algorithm", In Proc. IFAC Adchem, pp. 626-631, Jul. 2012.

[7] Shijoh. Vellayikot and M V Vaidyan, "Development of Nonlinear Model and Performance Evaluation of Model based Controller and Estimator for the Efficient Control of Hybrid three tank System", Int. journal of computer applications, vol. 56 No 12, pp. 11-16, 2012.

[8] N. N. Nandola, S. Bharatiya., 'Hybrid system identification using a structural approach and its model based control: An experimental validation', Nonlinear Analysis: Hybrid Systems, vol. 3, issue 2, pp. 87-100, 2009.

[9] Shijoh V., M. V. Vaidyan, "Development of generic dynamic nonlinear model for autonomous hybrid system and design of inverse dynamics controller and derivative free state estimator in presence of uncertainties", In Proc. IEEE iMac4s, India, Mar. 2013

[10] N. Barhoumi, F. Msahli, M. Djemaï, K. Busawon, "Observer design for some classes of uniformly observable nonlinear hybrid systems", Nonlinear Analysis: Hybrid Systems, Vol. 6, Issue 4, pp. 917929,

[11] W. Wanga, L. Lia, D. Zhoua, K. Liub, "Robust state estimation and fault diagnosis for uncertain hybrid nonlinear systems", Nonlinear Analysis: Hybrid Systems vol. 1, issue 1, pp. 2-15, 2007

[12] S. J. Julier and J. K. Uhlmann, "A new extension of the Kalman filter to nonlinear systems", Proc. AeroSense: $11^{\text {th }}$ Int. Symp. Aerospace/Defense Sensing, Simulation and Controls, pp.182 -193 1997

[13] Andrew. H. Jazwinski: "Stochastic Processes and Filtering Theory" Academic Press, New York 1970.

[14] R E. Kalman "A new approach to linear filtering and prediction problems". ASME-Journal of Basic Engineering, pp. 35- 45, 1960.

[15] R. Van der Merwe, E.A Wan, "The square-root unscented Kalman filter for state and parameterestimation," In proc. of IEEE International Con- 
ference ICASSP '01, vol. 6, no., pp. 3461-3464, 2001

[16] Y. Hao, Z. Xiong, F. Sun, X. Wang, "Comparison of Unscented Kalman Filters," In Proc. ICMA, pp. 895899, 5-8 Aug. 2007

[17] Z. Xie, J. Feng, "Real-time nonlinear structural system identification via iterated UKF", Mechanical systems and signal processing, vol. 28, pp. 309-322, 2012.

[18] V. A. Bavdekar, A. P. Deshpande, S. C. Patwardhan, "Identification of process and measurement noise covariance for state and parameter estimation using extended Kalman filter", Journal of Process Control, vol. 21, Issue 4, pp. 585-601, April 2011

[19] N. Orani, A. Pisano, M. Franceschelli, A. Giua, E. Usai, "Robust reconstruction of the discrete state for a class of nonlinear uncertain switched systems", Nonlinear Analysis: Hybrid Systems, vol. 5, Issue 2, pp. 220-232, 2011.

[20] J. Prakash, S. C. Patwardhan, S. L. Shah, "State estimation and nonlinear predictive control of autonomous hybrid system using derivative free state estimators", Journal of process control, vol. 20, Issue 7, pp. 787-799, August 2010.

[21] Jagadeeshan P., Sachin C. P., Sirish L. S, "Design and Implementation Fault Tolerant Model Predictive Control Scheme on a Simulated Model of ThreeTank Hybrid System", Conference on control and fault tolerant systems, France, pp. 173-178, 2010

[22] V. Shijoh, M. V. Vaidyan, and Varghese M. V., "Computer aided implementation of estimator based inverse dynamics controller for the control of nonmeasurable state variables of an autonomous hybrid system", LNSE, vol. 1, no. 3, pp. 284-288, 2013.

[23] Shijoh V., M. V. Vaidyan, "ANN approach for state estimation of hybrid systems and its experimental validationcom", Matematical problems in engineering, vol. 2015, Article ID 38232, pages 13.

[24] Shijoh V., M. V. Vaidyan, "Estimator based controllers for hybrid systems with uncertainties - an experimental validation", In proc. of ICACCI, Eranakulam, India, pp. 1044-1051, Aug. 2015.

[25] [25] Kumar, S., Prakash, J. and Kanagasabapathy, P. "A critical evaluation and experimental verification of extended kalman filter, unscented kalman filter and neural state filter for state estimation of three phase induction motor", Applied Soft Computing, vol. 11, Issue 3, pp. 3199-3208, 2011.

[26] [26] A. G. Parlos, S. K. M. and Amir F. Atiya, “An algorithmic approach to adaptive state filtering using recurrent neural networks", IEEE trans. on neural networks, vol. 12, no. 6, 2001, pp. 1411-1432.

[27] [27] E. Manitsas, R. Singh, B. C. Pal, and Goran Strbac, "Distribution System State Estimation Using an Artificial Neural Network Approach for Pseudo Measurement Modeling", IEEE trans. on power systems, vol. 27, no. 4, Nov. 2012.
[28] M. Jazaeri and M. T. Nasrabadi, "A new fast and efficient artificial neural network based state estimator incorporated into a linear optimal regulator for power system control enhancement", electric power components and systems, 43(6), 644-655, 2015.

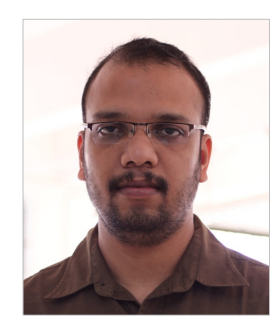

Shijoh Vellayikot is currently is a Research scholar in the Department of Electrical Engineering, National Institute of Technology, Calicut, India. $\mathrm{He}$ received B.Tech in Electrical and Electronics Engineering from Calicut University, Kerala, India and M. E. in Instrumentation Engineering from MIT Campus, Anna University, Chennai, India. His research interests include Process modeling and control, State estimation, and advanced model based control strategies.

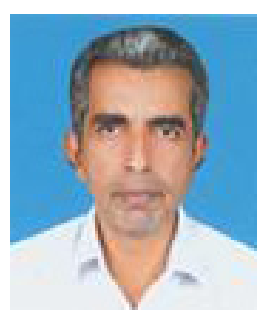

M. V. Vaidyan has obtained B.Sc. and M.Sc. degrees in engineering from University of Calicut. Presently he is professor in Electrical Engineering Department, National Institute of Technology, Calicut, India. His research interests spans around system identification, embedded systems, computer controlled systems, model reference adaptive systems, model predictive control, medical cybernetics, FPGA based system design etc. 